\title{
A MICROMACHINED RESONANT MAGNETIC FIELD SENSOR
}

\author{
Thierry C. Leïchlé, Martin von Arx and Mark G. Allen \\ School of Electrical and Computer Engineering \\ Georgia Institute of Technology \\ Atlanta, GA 30332-0250 \\ E-mail: leichle@atlas.mirc.gatech.edu: Fax: (404) 894-2776
}

The measurement of the direction and amplitude of magnetic fields is widely used in many applications. A variety of micromachined structures have been developed to perform this sensing function, including Hall-effect devices and fluxgate magnetometers. In general, there is a tradeoff between power consumption and sensitivity of these devices. For example, micromachined fluxgate magnetometers capable of measuring the direction of the magnetic field of the earth (e.g., 'micromachined compasses') require power consumption levels that are prohibitive for low power, portable applications such as watches.

The work presented here focuses on a resonant approach to the sensing of magnetic field amplitude and/or direction. The motivation for this approach is twofold: (1) resonant structures can be fabricated that require very little actuation power; and (2) the resonant frequency of the structure can be made exquisitely sensitive to small stresses, e.g., the stress caused by the interaction of an external magnetic field with the structure. A typical resonator structure consists of a freestanding plate attached to two anchor pads by means of two beams. It is excited electrostatically by applying an $\mathrm{AC}$, superposed with a DC, voltage between the plate and a bottom electrode located on the substrate. The resonant frequency of the system shifts when a magnetic field is applied. The interaction between the applied field and the magnetization induced in the material causes a change in the stiffness of the beams, thereby changing the resonant frequency of the device.

As an illustration of this concept, a simple device has been designed, fabricated, and tested. The fabrication of the device is based on conventional UV photolithography, micromolding and electroplating of ferromagnetic alloys. The fabrication sequence is shown in Figure 1. It starts with the deposition of a $300 \oplus$ titanium / $2000 \oplus$ aluminum layer on a glass substrate. This metal layer is pattemed using photolithography and wet-etched to create the bottom electrode. An insulator ( $10 \mu \mathrm{m}$ of P12611 polyimide) is then spun and cured on top of the electrodes. A seed-layer of 200 $\oplus$ titanium / $3000 \oplus$ copper is evaporated on top of the polymer. Photoresist (AZ 4620) is then spun and processed in order to create a $30 \mu \mathrm{m}$ thick mold. A $5 \mu \mathrm{m}$ thick copper layer is first electrodeposited in the mold as a sacrificial layer. A $20 \mu \mathrm{m}$ thick nickel (80\%) - iron (20\%) magnetic alloy is electroplated on top of the copper to create the resonating structure. The photoresist mold is dissolved and the copper sacrificial layer is then selectively and isotropically removed. Since the pads and anchors of the device are larger than the support beams or the resonating portion, no patteming of the sacrificial layer is required as long as the wet etch is stopped as soon as the moving plate is released. The glass substrate is then diced and the devices are individually packaged for testing. Figures 2 and 3 show an optical photomicrograph and a scanning electron micrograph of completed devices, respectively.

Functional devices have been successfully fabricated and tested. The device has been tested in open loop in order to determine the natural resonance frequency (typically on the order of $1 \mathrm{kHz}$ ). The device has been placed inside an electromagnet that generated the magnetic field in the plane of the resonator. Since the as-fabricated device was a one-port resonator, the two electrodes have been used to excite the resonator as well as to detect the resonant frequency by measuring the capacitance change between the excitation plates as a finction of frequency. A gain-phase analyzer generated the signal that was applied, after amplification, to the resonator. Assessment of the frequency dependence of the magnitude and phase of the resultant displacement current by means of the gain-phase analyzer provided the sensor resonant frequency. This resonant frequency was then measured as a function of incident magnetic field amplitude and direction.

Figure 4 shows the variation of the resonant frequency of the device (in $\mathrm{Hz}$ ) as a function of the applied magnetic field (in Oersted) when the direction of the field is parallel to the plate. As expected, due to the symmetry of the device an absolute-value type response is seen, with minimum resonant frequency at the point of zero applied magnetic field. Resolutions of 20 Oersted in amplitude of the field have been achieved. The dependence of the resonant frequency on rotation angle between applied magnetic field and the device was also studied, and is given in Figure 5 for a magnetic field excitation of 500 Oersted. An angular resolution of $20^{\circ}$ in the direction of the field has been achieved. Although this unoptimized and non-integrated device has not yet proven itself to be of sufficient sensitivity to sense the magnetic field of the earth, the functionality of the sensor has been demonstrated, and the sensing principle should allow for scaling of the sensitivity into this regime without large increases in power consumption. 


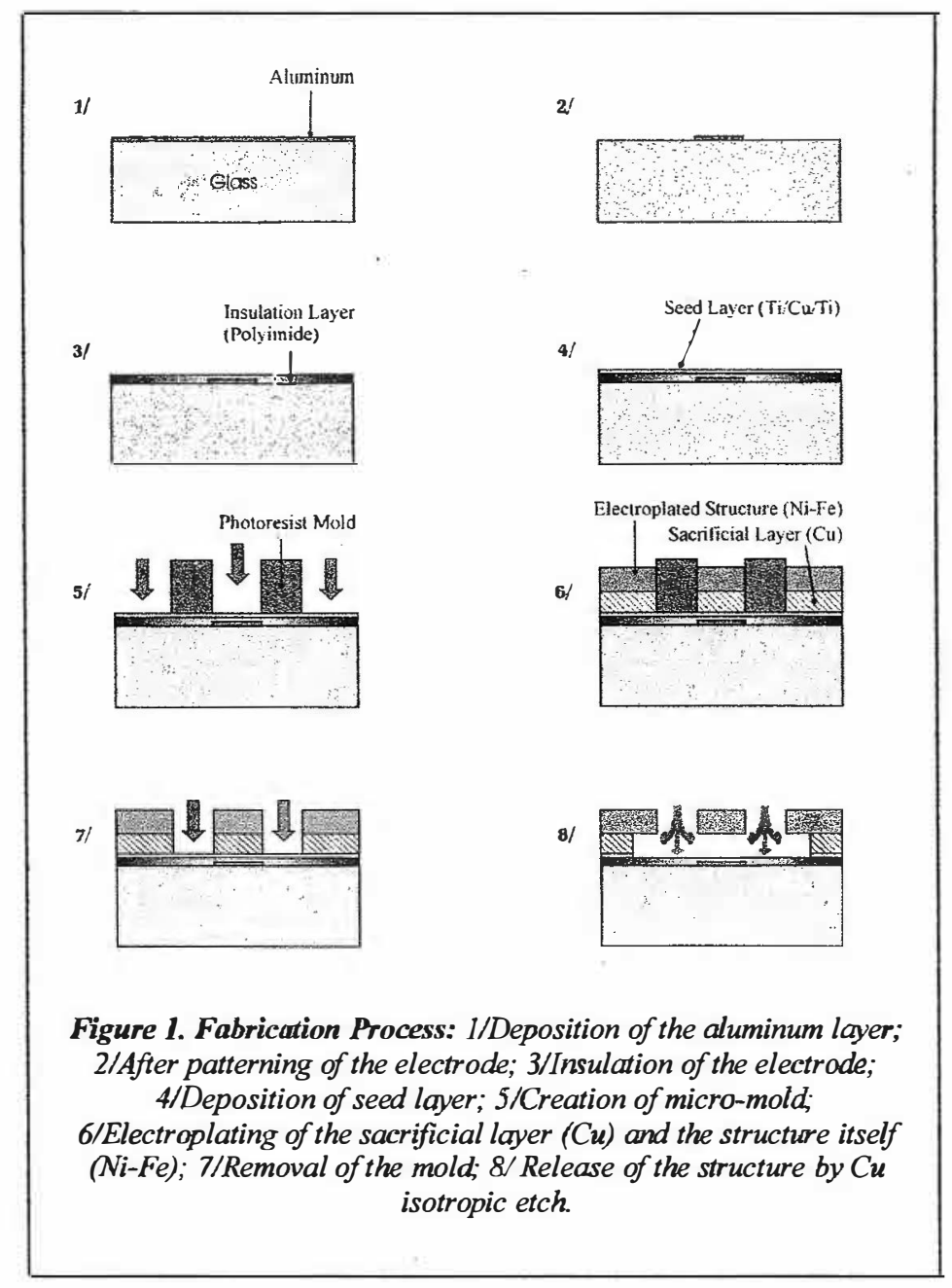

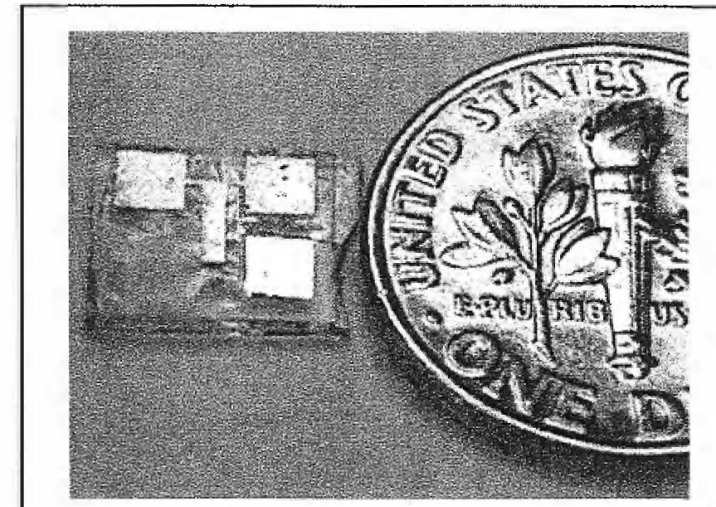

Figure 2. Photograph of a fabricated device and a dime.

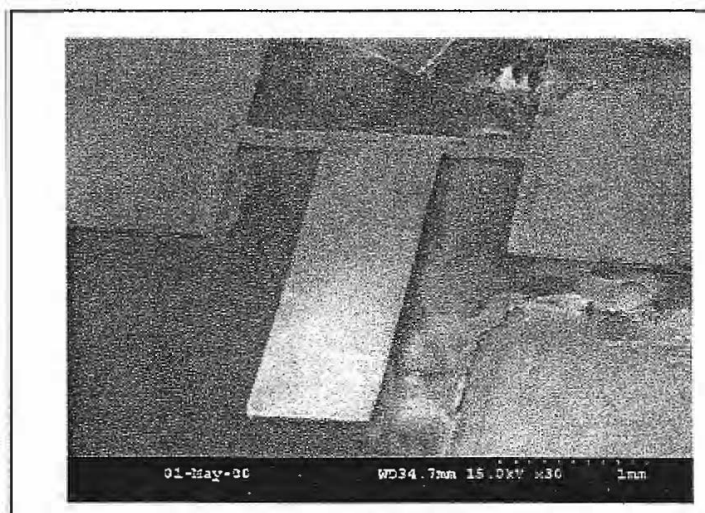

Figure 3. SEM photograph of a fabricated device.

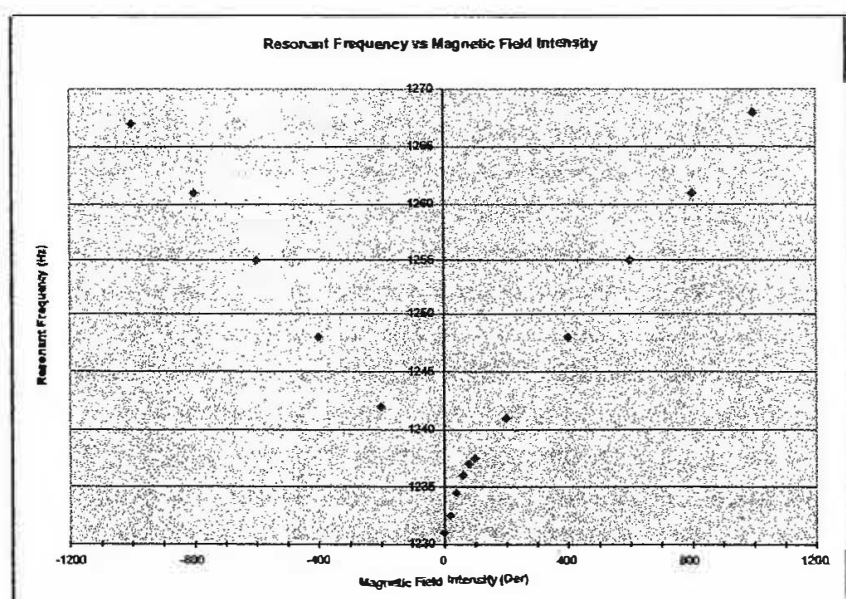

Figure 4. Change of the resonant frequency of the device as a function of the magnetic field intensity (when the direction of the field is porallel to the resonator plate).

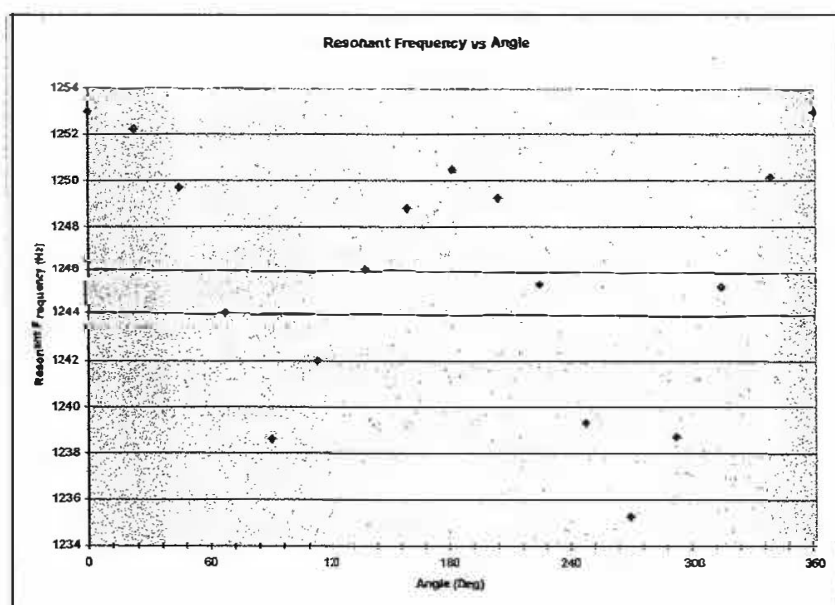

Figure 5. Change of the resonant frequency of the device as a function of the direction of the magnetic field (the intensity of the field is 500 Oersted). 